\title{
A Hybrid Algorithm for Privacy Preserving in Data Mining
}

\author{
Sridhar Mandapati \\ Dept. of Computer Applications, R.V.R \& J.C College of Engineering, Guntur, India \\ E-mail:mandapati_s@yahoo.com \\ Dr. Raveendra Babu Bhogapathi \\ Dept. of Computer Science and Engineering, VNR VJIET, Hyderabad, India \\ E-mail: rbhogapathi@yahoo.com
}

Ratna Babu Chekka

Dept. of Computer Science and Engineering, R.V.R \& J.C College of Engineering, Guntur, India E-mail: chekka.ratnababu@gmail.com

\begin{abstract}
With the proliferation of information available in the internet and databases, the privacypreserving data mining is extensively used to maintain the privacy of the underlying data. Various methods of the state art are available in the literature for privacypreserving. Evolutionary Algorithms (EAs) provide effective solutions for various real-world optimization problems. Evolutionary Algorithms are efficiently employed in business practice. In privacy-preserving domain, the existing EA solutions are restricted to specific problems such as cost function evaluation. In this work, it is proposed to implement a Hybrid Evolutionary Algorith m using Genetic Algorith m (GA) and Particle Swarm Optimization (PSO). Both GA and PSO in the proposed system work with the same population. In the proposed framework, k-anonymity is accomplished by generalization of the original dataset. The hybrid optimization is used to search for optimal generalized feature set.
\end{abstract}

Index Terms - Privacy-Preserving Data Mining (PPDM), Evolutionary Algorithms (EAs), Swarm Intelligence, Genetic Algorithm (GA), Particle Swarm Optimization (PSO), Adult Dataset

\section{Introduction}

Technology advances in hardware and software have led to increased storage capabilities. With the proliferation of information about individual's personal data available in the internet and databases, data mining is considered as a threat to privacy of data. The privacypreserving data mining is extensively used to maintain the privacy of the underlying data. Privacy-preserving data mining (PPDM) algorith ms are so constructed that the confidential data which is mined is not revealed to the user running the algorithm. The main concerns of
PPDM is that sensitive raw data like names, addresses are modified from the original database, so that the users of the data will not be able to compromise another person's privacy. And also, sensitive knowledge obtained from mining which can compromise data privacy must be excluded. Thus, privacy preservation is to be integrated at two levels, users' personal information and information relating to their collective activity. The former is known as individual privacy preservation and the latter as collective privacy preservation [1].

Privacy preserving of data must safeguard from divulging sensitive data during publication of individual data. To maintain privacy, a number of techniques have been proposed for modify ing or transforming the data. To avoid data misuse, the data is anonymized. Many data mining techniques are modified to ensure privacy. The techniques for PPDM are based on cryptography, data mining and information hiding [2]. In general, statistics-based and the crypto-based approaches are used to tackling PPDM. In the statistics-based approach, the data owner's sanitize the data through perturbation or generalization before publishing. Knowledge models such as decision trees are used on the sanitized data. The advantage of statistics-based approach is that it efficiently handles large volume of datasets [3]. In the crypto-based PPDM approach, data owners have to cooperatively implement specially designed data mining algorithms [4]. Though these algorithms achieve verifiable privacy protection and better data mining performance, it suffers from performance and scalability issues [5].

In recent years, privacy preserving data for a single database has been extensively studied [6]. Data anonymization transforms a dataset to uphold privacy using methods such as k-anonymity using generalization or suppression techniques, so that individually identifiable information is masked. K- 
Anonymity transforms data to equivalence classes and each class has a set of $\mathrm{K}$ - records indistinguishable from each other [7-9]. Problems with this approach were remedied using techniques like 1 -diversity and $t$ closeness [10, 11].

The common methodology of k-anonymity used is generalization, where certain values are replaced with less specific but semantically consistent values. Otherwise, some of the values are suppressed. The problem of discovering optimal k-anonymous datasets using generalization or suppression has been proved to be NP-hard [12, 13]. Minimum data loss can be achieved by optimizing an aggregated value over all features and records. The Evolutionary Algorithms (EA) based on swarm intelligence used simple entities with limited memory evolving into increasingly better solutions. The efficient swarm-based data mining approaches usually are some kind of hybrid approach; such as combining a swarm intelligence technique with some orthodox optimization technique, such as the PSO-based clustering technique where the solution is obtained by k-means clustering [14] or combine several swarm-based approaches, such as the PSO/ACO technique [15].

Particle Swarm Optimization (PSO) and Genetic Algorithm (GA) are population based heuristic search technique, popularly used to solve the optimization problems modelled on the basis of EA. In standard PSO, a particle which can stagnate due to the non-oscillatory route and also suboptimal solutions are obtained due to premature converging. Minimizing or maximizing an objective function based on the constraints imposed is the basic problem faced during optimization. Global optimization is absolutely the best set of admissible conditions achievable for an objective under given constraints. Hybrid algorithms incorporating GA are proposed to overcome the limitations of PSO. These hybrid algorithms have the advantages of PSO with those of GA.

The difference between PSO and GA is the capability to control convergence. In GA, the crossover and mutation rates affect the convergence, but are not comparable to the level of control accomplished through controlling of the inertia weight in PSO. The swarm's convergence increases radically with the decrease in inertia weight. The drawback being that the PSO converges [16] to stable point, which is not essentially maximum. To prevent the occurrence, position update of the global best particles is changed. The hybrid mechanism of GA updates the genetic operator's crossover and mutation helps in enhancing the PSO. The crossover operation swaps information between two particles, thus enhancing the ability to fly through new search area [17]. While mutation increases the diversity of the population, and helps avoid the local maxima.

In this work, it is proposed to implement a Hybrid Evolutionary Algorithm using Genetic A lgorithm (GA) and Particle Swarm Optimization (PSO). Both GA and PSO in the proposed system work with the same population. In the proposed framework, k-anonymity is accomplished by generalization of the orig inal dataset and the hybrid optimization is used to search for optimal generalized feature set. The paper is organized as follows: Section 2 reviews some related works in the literature, section 3 details the methods, section 4 gives the results and discussions and section 5 concludes the paper.

\section{Related Works}

Bayardo, et al., [18] proposed an optimization algorith $\mathrm{m}$ for $\mathrm{k}$-anonymization. The proposed method searches the space of possible anonymization and forms strategies to reduce computation. The census data was used for evaluation and experiments show that the proposed method achieves optimal k-anonymizations using a wide range of $\mathrm{k}$. The effects of different coding approaches and quality of anonymization and performance were also investigated. Real census data experiments demonstrated that the proposed algorithm could locate optimal k-anonymizations under two representative cost measures and a wide range of $\mathrm{k}$.

Sakuma et al [19] proposed a protocol for a local search and a genetic algorithm for the distributed traveling sales man problem (TSP). In the distributed TSP, the cost function information is possessed by distributed parties and is not disclosed to each other. The proposed method is a combination of genetic algorithms and a cryptographic technique, called the secure multiparty computation, which solves the distributed TSP. The privacy preserving LS that adopts 2-opt as neighbourhood and a privacy preserving GA that adopts EAX as crossover and CCM as selection method based on a protocol that solves private scalar product comparis on.

Dehkordi et al [20] introduced a new multi-objective method for hiding sensitive as sociation rules using GAs. The objective of the proposed method is to fully support the security of database and to keep the utility and certainty of mined rules at the highest level. In the proposed framework, a pre-sanitization process called Dataset Pre-Sanitization Process (DPSP) is implemented which selects transaction(s) and item(s) in each transaction to be changed for concealing the association rules. Four sanitization strategies were proposed with different criterion.

Matatov et al [21] proposed an approach, data mining privacy by decomposition (DMPD), for achieving kanonymity by partitioning the original dataset into several projections with each one of them maintaining k-anonymity. Rejoining the projections resulted in a table which still maintained the k-anonymity. Each projection is used to train a classifier and consequently, a new instance is classified by combining the classifications of all classifiers. Guided by classification 
accuracy and k-anonymity constraints, the proposed algorith $m$ uses a genetic algorith $m$ to search for optimal feature set partitioning. DMPD was evaluated using ten separate datasets and its classification performance was compared with other k-anonymity-based methods. The proposed DMPD performs better than existing kanonymity-based algorithms .

\section{Methodol ogy}

\subsection{Adult Dataset}

UCI Machine Learning Repository provides the 'Adult' dataset used for evaluation. It contains 48,842 instances, including categorical and integer attributes from 1994 Census information. It has about 32,000 rows with 4 numerical columns, the column which includes age $\{17-90\}$, fnlwgt $\{10000-1500000\}$, hrsweek $\{1-100\}$ and edunum $\{1-16\}$. The age column and native country are anonymized using kanonymization. Table 1 shows the original attributes of the Adult dataset.

Table 1: Attributes of the Adult Dataset

\begin{tabular}{|c|l|c|}
\hline Age & native-country & Class \\
\hline 39 & United-States & $<=50 \mathrm{~K}$ \\
\hline 50 & United-States & $<=50 \mathrm{~K}$ \\
\hline 38 & United-States & $<=50 \mathrm{~K}$ \\
\hline 53 & United-States & $<=50 \mathrm{~K}$ \\
\hline 28 & Cuba & $<=50 \mathrm{~K}$ \\
\hline 37 & United-States & $<=50 \mathrm{~K}$ \\
\hline 49 & Jamaica & $<=50 \mathrm{~K}$ \\
\hline 52 & United-States & $>50 \mathrm{~K}$ \\
\hline 31 & United-States & $>50 \mathrm{~K}$ \\
\hline 42 & United-States & $>50 \mathrm{~K}$ \\
\hline
\end{tabular}

\subsection{K-Anonymity}

In k-anonymity, the data is transformed to equivalence classes where each class has a set of krecords that differs from others [22]. Generalization \& suppression are used to reduce the granularity representation of the pseudo-identifiers techniques. The attributed values are generalized to a range so as to reduce the granularity (for example, date of birth generalized as year of birth) and it also reduces identification risk. The value of the attribute is removed completely to reduce the identification risk with public records (suppression). The k-anonymity is a good technique because of its simplicity in definition and also many algorithms are available to process the anonymization [23, 24].

\subsection{Genetic Algorithm (GA)}

In Genetic Algorithm (GA), a group of individuals called chromosomes forms the population that represents a complete solution to a defined problem $[25$, 26]. Each chromosome is encoded using a sequence of 0 s or 1s. The GA begins using a randomly generated set of individuals as population. In each iteration, a new population is generated which replaces all of members of the population. Though, certain number of the best individuals is kept from each generation and is copied with the new generation (this approach known as elit is $\mathrm{m}$ ). The best chromosome in the population is used to generate the next population. Based on the fitness functions, the population will transform into the future generation.

On evaluation of population's fitness, fittest chromosomes are selected for reproduction. Lower fitness chromosomes or poor chromosomes might be selected in very less numbers or not at all. There are popular selection methods such as "Roulette-Wheel" selection, "Rank" selection and "Tournament" selection. In this study, Tournament selection is used wherein two chromosomes are chosen randomly from the population. First, for a predefined probability $p$, the more fit of these two is selected and with the probability (1-p) the other chromosome with less fitness is selected [26].

The crossover operation in GA combines two chromosomes together to produce new offspring (child). Crossover occurs only with crossover probability. Chromosomes remain the same when not subjected to crossover. The idea behind crossover is considering new solutions and exploiting of the old solutions. As fittest chromosomes are selected more, good solutions are carried to the next generation. In this study, singlepoint crossover has been applied to produce new offspring for that a high value of crossover probability is used (between 0.80 and 0.90).

Due to crossover operation, the new generation will contain only the character of the parents. This can lead to a problem saturation of find ing a better population as no new genetic material is introduced in the offspring. Mutation operator introduces new genetic patterns into the new chromosomes. The new sequence of genes due to mutation may or may not produce desirable features in the new chromosome. The new mutated chromosome is kept if the fitness is better than the general population.

\subsection{The Particle Swarm Optimization (PSO)}

The Particle Swarm Optimization (PSO) algorith $\mathrm{m}$ is an adaptive algorith $m$ made of population of individuals (commonly referred to as particles), adapting through returning stochastically toward previous successful regions [27, 28]. The two primary operators in PSO are Velocity update and Position update. During iteration, particle is accelerated toward the particles in the previous best position and the global best position. A new velocity value is updated for each particle at 
iterations and the updated velocity is based on its current velocity, distance from its previous best position, and distance from its global best position. This is utilized to calculate the next position of the particle in search space. The procedure stops either on iteration of a specific number of times, or till a minimum error is obtained [29, 30].

PSO begins with a group of random particles or solutions and searches for optima through updating of generations. The two "best" values, pbest and gbest, of the particle are updated in each iteration. 'pbest' is the best solution (fitness) achieved till then and ' $g$ best' value is the best value obtained till then by any particle in the population. PSO is computationally simple as it requires only primitive mathe matical operators. Particle positions and velocities are assigned randomly in the beginning of the algorithm. PSO updates all velocities and positions of all the particles iteratively as follows:

$$
\begin{aligned}
& v_{i}^{d}=w v_{i}^{d}+c_{1} r_{1}\left(p_{i}^{d}-x_{i}^{d}\right)+c_{2} r_{2}\left(p_{g}^{d}-x_{i}^{d}\right) \\
& x_{i}^{d}=x_{i}^{d}+v_{i}^{d}
\end{aligned}
$$

where

$v_{i}^{d}$ - new velocity of the $\mathrm{i}^{\text {th }}$ particle computed based on the particle's previous velocity, distance between the previous best position and current position and distance between the best particle of the swarm

$$
\begin{aligned}
& \mathrm{d} \text { - number of dimensions, } \\
& \mathrm{i} \text { - size of the population, } \\
& \mathrm{w} \text { - inertia weight, } \\
& \mathrm{r} 1 \text { and } \mathrm{r} 2 \text { are random values in the range }[0,1] \\
& \mathrm{c}_{1}, \mathrm{c}_{2} \text { are positive constants, } \\
& x_{i}^{d}-\text { the new position of the particle. }
\end{aligned}
$$

In classical PSO, the particles tend to get trapped in the local optimum in the gbest region if gbest is far away from the global optimum. To overcome this, the particles are made to fly through a larger search space and pbest position of a particle is updated based on the pbest position of all the particles in the swarm. This improves the diversity of the swarm and local optimum is avoided. The updating velocity of the particle is given by:

$$
V_{i}^{d}=w^{*} v_{i}^{d}+c * \operatorname{rand}_{i}^{d} *\left(\operatorname{pbest}_{f i(d)}^{d}-x_{i}^{d}\right)
$$

$$
\text { Where } f_{i}=\left[f_{i}(1), f_{i}(2), \ldots, f_{i}(d)\right] \text { refers to the }
$$
pbest that the particle i used and pbest $_{f i(d)}$ is the dimension of particles pbest. Two particles are selected randomly and one of it whose velocity is updated is left out. To update the velocity, the fitness value of the individual particles pbest is compared to select the best dimension.

\subsection{Hybrid GA-PSO}

Cooperative search is a type of parallel algorithms, where several search algorithms are run in parallel to solve the optimization problem. As the search algorithms may be different, cooperative search technique is viewed as a hybrid algorithm [31]. In this work, it is proposed to implement a Hybrid Evolutionary Algorithm using Genetic Algorithm (GA) and Particle Swarm Optimization (PSO). Both GA and PSO in the proposed system work with the same population. Initially, Ps individuals which form the population are generated randomly. They can be considered chromosomes in GA, or as particles in PSO. After initialization, new next generation individuals are created by enhancement, crossover, and mutation operations. The architecture of the proposed hybrid algorithm is given below.

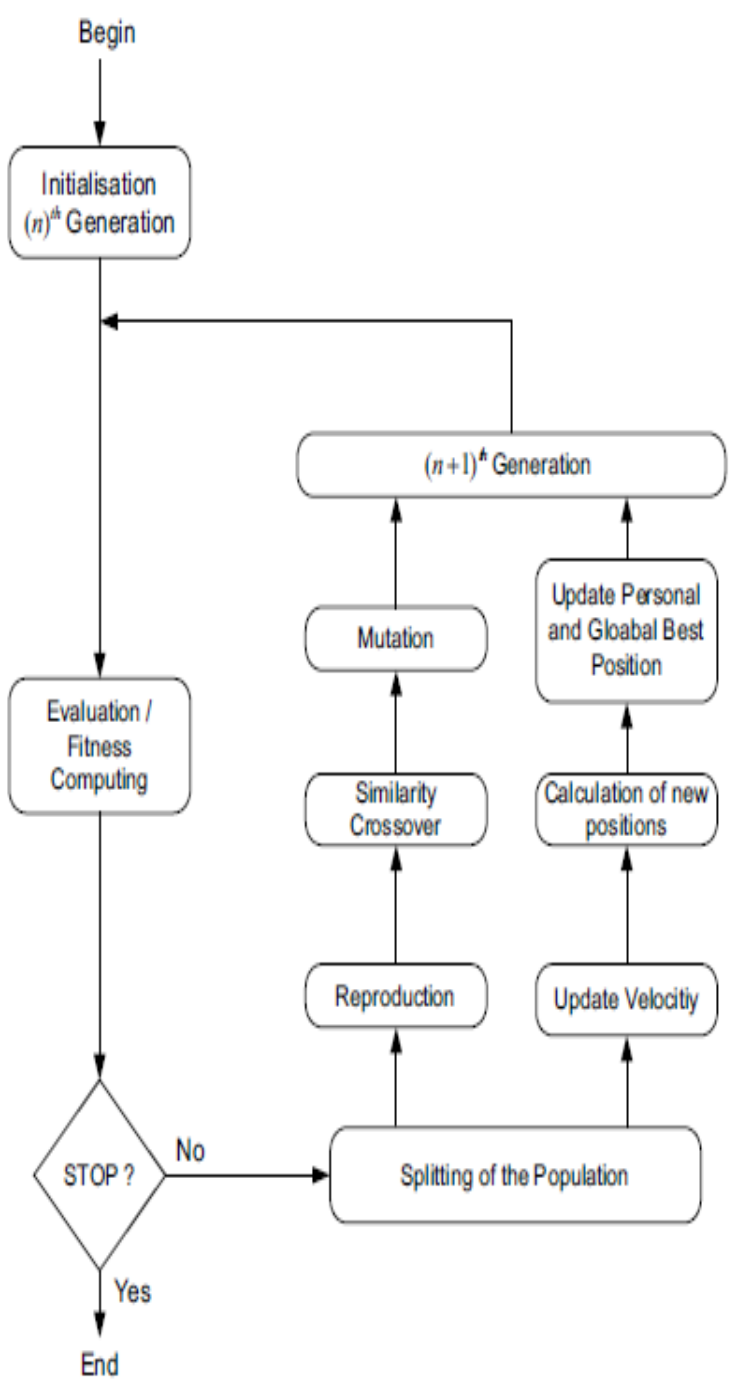

Fig. 1: Flowchart of the Proposed Hybrid Algorithm incorporating GA and PSO 


\section{Results and Discussion}

The generalization depends on the type of data; it can either be categorical or numeric. The generalization of the categorical data (gender, work, zip code) is described by a taxonomy tree as seen in Figure 2. The Figure shows an example for generalization of continuous data used in this work.

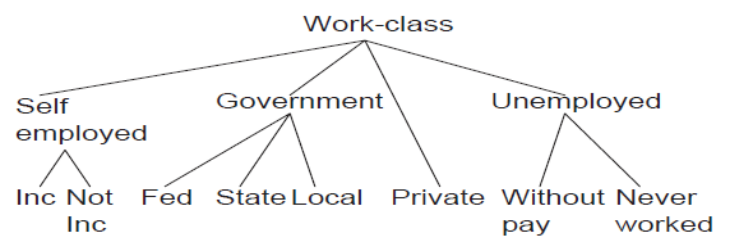

Fig. 2: Examplefor Generalization of continuous data as a taxonomy tree

For generalization of numeric data (age, income) is obtained by discretization of its values into a set of disjoint intervals. Various levels of discretization defined, for numeric data of age, the set of intervals:

$$
\begin{aligned}
& \{(0,10),(10,20),(20,30), . .\} ; \\
& \{(0,20),(20,40),(40,60), . .\} ; \\
& \{(0,30),(30,60),(60,90), . .\} \text { are valid. }
\end{aligned}
$$

Experiments are conducted for different levels of kanonymity $(5,10, \ldots, 45,50)$. Hybrid algorith $m$ is used to find the optimal generalization feature set. Table 2 shows the parameter used for GA in this study. Following Figures and Tables give the results of classification, precision and recall for class label income. The precision and recall is shown for value greater than $50 \mathrm{~K}$ and less than or equal to $50 \mathrm{~K}$.

Table 2: The Proposed Hybrid Algorithm Parameters

\begin{tabular}{|l|c|}
\hline Initial population size & 25 \\
\hline Maximum generations & 20 \\
\hline Number of epochs & 500 \\
\hline Momentum optimization & $\begin{array}{c}\text { Lower bound } 0.5 \\
\text { Upper bound } 1.0\end{array}$ \\
\hline Step size optimization & $\begin{array}{l}\text { Lower bound } 0.1 \\
\text { Upper bound } 0.5\end{array}$ \\
\hline Encoder mechanism & Roulette \\
\hline Cross over & Single point \\
\hline Cross over probability & 0.9 \\
\hline Mutation & Uniform \\
\hline Mutation probability & 0.01 \\
\hline
\end{tabular}

Table 3: Classification Accuracy for different levels of k-anonymity

\begin{tabular}{|c|c|}
\hline k-anonymity level & Classification accuracy \\
\hline $\mathrm{K}=50$ & 0.832500717 \\
\hline $\mathrm{K}=45$ & 0.833135416 \\
\hline $\mathrm{K}=40$ & 0.836083698 \\
\hline $\mathrm{K}=35$ & 0.840362803 \\
\hline $\mathrm{K}=30$ & 0.847242128 \\
\hline $\mathrm{K}=25$ & 0.855759387 \\
\hline $\mathrm{K}=20$ & 0.862454445 \\
\hline $\mathrm{K}=15$ & 0.870582695 \\
\hline $\mathrm{K}=10$ & 0.875619344 \\
\hline $\mathrm{K}=5$ & 0.880389828 \\
\hline
\end{tabular}

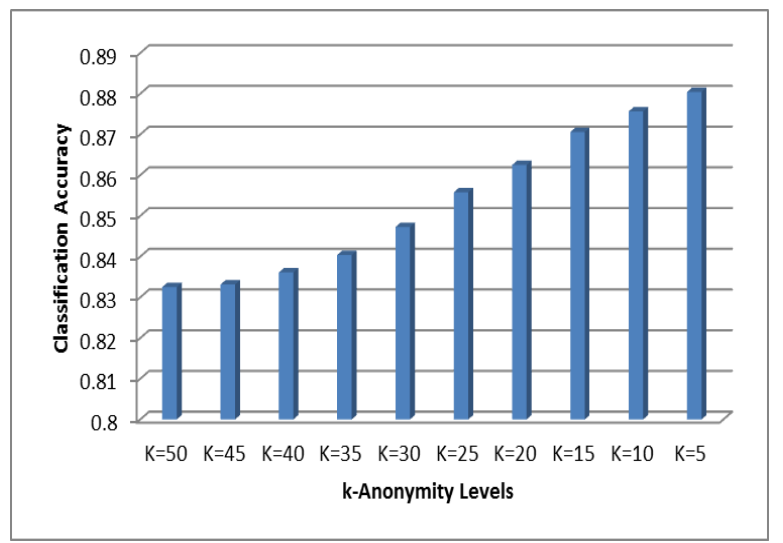

Fig. 3: Classification Accuracy for different levels of k-anonymity

It is observed from Figure 3, that the classification accuracy decreases with the increase in k-anonymity level. Figure 4 and 5 show the precision and recall for class label income greater than $50 \mathrm{k}$ and less than or equal to $50 \mathrm{k}$ respectively.

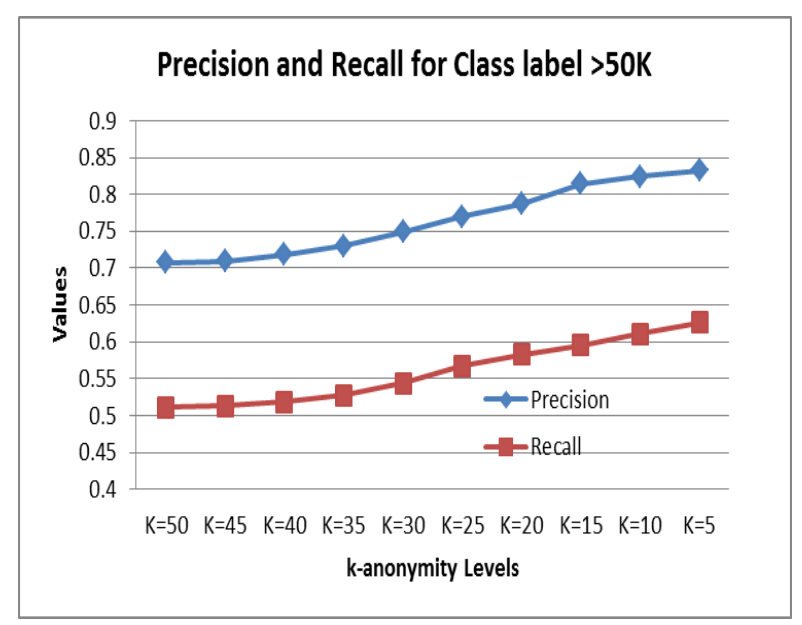

Fig. 4: Precision and Recall for different levels of k-anonymity for class label $>50 \mathrm{~K}$

I.J. Intelligent Systems and Applications, 2013, 08, 47-53 


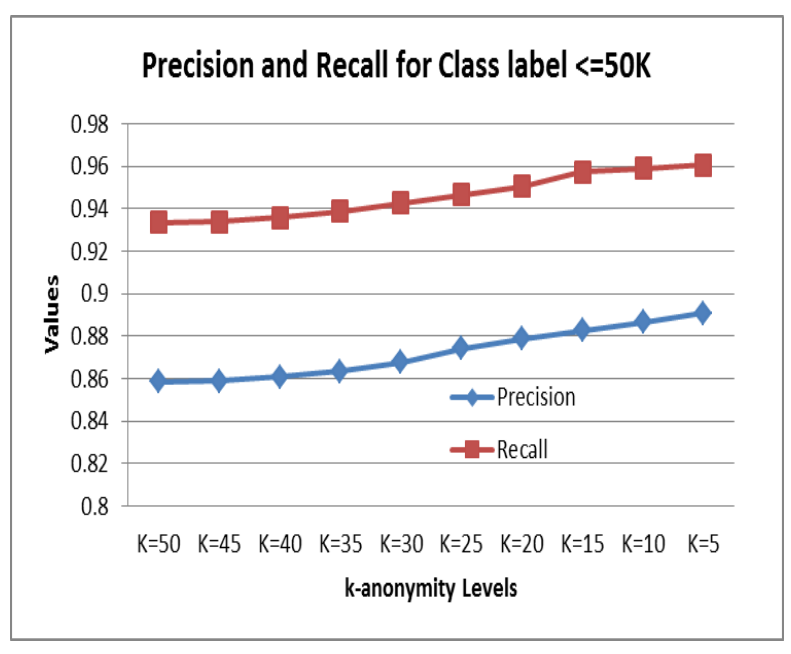

Fig. 5: Precision and Recall for different levels of k-anonymity for class label $<=50 \mathrm{~K}$

\section{Conclusion}

Existing Evolutionary Algorithm (EA) solutions in privacy-preserving domain mainly deals with specific problems such as cost function evaluation. In this work, it is proposed to implement a Hybrid EA using Genetic Algorithm (GA) and Particle Swarm Optimization (PSO). Both GA and PSO complement each other to provide global optimization. In the proposed frame work, k-anonymity is accomplished by generalization of the original dataset. The hybrid optimization is used to search for optimal generalized feature set. Experiments were conducted for different levels of $\mathrm{k}$-anonymity and the results obtained are satisfactory.

\section{Acknowledgments}

We profusely thank our most generous management for allowing us to make use of the infrastructure on the campus and the support extended for the fulfilment of the research paper. The authors would like to thank the anonymous reviewers for their careful reading of this paper and for their helpful comments.

\section{References}

[1] Xinjing Ge and Jianming Zhu, (2011), Privacy Preserving Data Mining, New Fundamental Technologies in Data Mining.

[2] Agrawal R., Srikant R. Privacy-Preserving Data Mining. Proceedings of the ACM SIGMOD Conference, 2000.

[3] Malin, B., Benitez, K., \& Masys, D. (2011). Never too old for anonymity: a statistical standard for demographic data sharing via the HIPAA Privacy Rule. Journal of the A merican Medical Informatics Association, 18(1), 3-10.
[4] Singh, M. D., Krishna, P. R., \& Saxena, A. (2010, January). A cryptography based privacy preserving solution to mine cloud data. In Proceedings of the Third Annual ACM Bangalore Conference (p. 14). ACM.

[5] Patrick Sharkey, Hongwei Tian, Weining Zhang, and Shouhuai Xu, 2008, Privacy-Preserving Data Mining through Knowledge Model Sharing, Springer-Verlag Berlin Heidelberg, pp. 97-115, 2008

[6] Pawel Jurczyk, Li Xiong, 2008, PrivacyPreserving Data Publishing for Horizontally Partitioned Databases, CIKM'08, October 2630USA., ACM 978-1-59593-991-3/08/10.

[7] Campan, A., \& Truta, T. (2009). Data and structural k-anonymity in social networks. Privacy, Security, and Trust in KDD, 33-54.

[8] Nergiz, M. E., Clifton, C., \& Nergiz, A. E. (2009). Multire lational k-anonymity. Knowledge and Data Engineering, IEEE Transactions on, 21(8), 11041117.

[9] Stokes, K., \& Torra, V. (2012, March). NConfusion: a generalization of k-anonymity. In Proceedings of the 2012 Joint EDBT/ICDT Workshops (pp. 211-215). ACM.

[10] Cao, J., Karras, P., Kalnis, P., \& Tan, K. L. (2011). SABRE: a Sensitive Attribute Bucketization and REdistribution framework for $t$-closeness. The VLDB Journal, 20(1), 59-81.

[11] Shi, P., Xiong, L., \& Fung, B. (2010, October). Anonymizing data with quasi-sensitive attribute values. In Proceedings of the 19th ACM international conference on Information and knowledge management (pp. 1389-1392). ACM.

[12] A. Meyerson, R. Williams, On the complexity of optimal k-anonymity, in: Proc. of the 23rd ACM SIGMOD-SIGCAT-SIGART Symposium, ACM, New York, NY, 2004, pp. 223-228.

[13] P.Samarati, Protecting respondents' identities in microdata release, IEEE Transactions on Knowledge and Data Engineering 13 (6) (2001) 1010-1027.

[14] Van der Merwe, D., \& Engelbrecht, A. P. (2003). Data clustering using particle swarm optimization. In IEEE congress on evolutionary computation (1) (pp. 215-220). New York: IEEE.

[15] Holden, N., \& Freitas, A. (2008). A hybrid $\mathrm{PSO} / \mathrm{ACO}$ algorith $\mathrm{m}$ for discovering classification rules in data mining. Journal of Artific ial Evolution and Applications, 2008, 11 pages.

[16] Van den Bergh F. and Engelbrecht A.P., 'A Cooperative Approach to Particle Swarm Optimization', IEEE Transactions on Evolutionary Computation, 2004, pp. 225-239. 
[17] Premalatha, K., \& Natarajan, A. M. (2009). Hybrid PSO and GA for global maximization. Int. J. Open Problems Compt. Math, 2(4), 597-608.

[18] Bayardo R. J., Agrawal R.: Data Privacy through Optimal k-Anonymization. Proceedings of the ICDE Conference, pp. 217-228, 2005.

[19] Sakuma, J., \& Kobayashi, S. (2007, July). A genetic algorithm for privacy preserving combinatorial optimization. In Proceedings of the 9th annual conference on Genetic and evolutionary computation (pp. 1372-1379). ACM.

[20] Dehkordi, M. N., Badie, K., \& Zadeh, A. K. (2009). A novel method for privacy preserving in association rule mining based on genetic algorithms. Journal of software, 4(6), 555-562.

[21] Matatov, N., Rokach, L., \& Maimon, O. (2010). Privacy-preserving data mining: A feature set partitioning approach. Information Sciences, 180(14), 2696-2720.

[22] P. Samarati and L. Sweeney. Protecting privacy when disclosing information: k-anonymity and its enforcement through generalization and suppression. Technical report, CMU, SRI, 1998.

[23] Lefevre, K., Dewitt, D., And Ramakrishnan, R. 2005. Incognito: Efficient full domain $\mathrm{k}$ anonymity. In SIGMOD.

[24] Zhong, S., Yang, Z., And Wright, R. N. 2005. Privacy-enhancing k-anonymization of customer data. In Proceedings of the International Conference on Principles of Data Systems (PODS).

[25] L. David, Handbook of Genetic Algorithms. New York: Van Nostrand Reinhold. 1991.

[26] D.E. Goldberg, Genetic Algorithms: in Search, Optimization, and Machine Learning. New York: Addison-Wesley Publishing Co. Inc. 1989.

[27] Qing Cao, Tian He, and Tarek Abdelzaher, uCast: Unified Connectionless Multicast for Energy Efficient Content Distribution in Sensor Networks, IEEE Transactions On Parallel And Distributed Systems, Vol. 18, No. 2, February 2007

[28] Latiff, N.M.A.; Tsimenidis, C.C.; Sharif, B.S., "Performance Comparison of Optimization Algorithms for Clustering in Wireless Sensor Networks," Mobile Adhoc and Sensor Systems, 2007. MASS 2007. IEEE International Conference on , vol., no., pp.1-4, 8-11 Oct. 2007

[29] Matthew Settles," An Introduction to Particle Swarm Optimization", 2005

[30] Eberhart, R. C., Shi, Y.: Particle swarm optimization: Developments, applications and resources, In Proceedings of IEEE International Conference on Evolutionary Computation, vol. 1 (2001), 81-86.
[31] El-Abd, M., \& Kamel, M. (2005). A taxonomy of cooperative search algorithms. Hybrid Metaheuristics, 902-902.

\section{Authors’ Profiles}

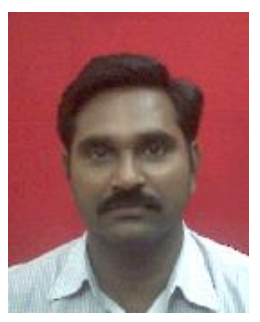

Sridhar Mandapati obtained his masters degree in Computer Applications from S.V University, Tirupathi. He is currently working as Associate Professor in the Department of Computer Applications at R.V.R. \& J.C College of Engineering, Guntur.

He has 14 years of teaching experience. At present he is pursuing Ph.D. from Acharya Nagarjuna University, Guntur. His areas of research interest include Data Mining, Information Security and Image Processing.

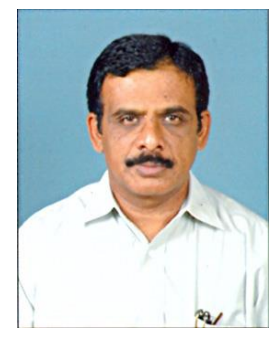

Dr. Raveendra Babu Bhogapathi obtained his Masters in Computer Science and Engineering from Anna University, Chennai. He received his Ph.D. in Applied Mathematics at S.V University, Tirupati. He is now working as Professor in the Department of Computer Science and Eng ineering, VNR VJIET, Hyderabad. He has 27 years of teaching experience. He has more than 55 International and National publications to his credit. His research areas of interest include Data Mining, Image Processing, Pattern Analysis and Information Security.

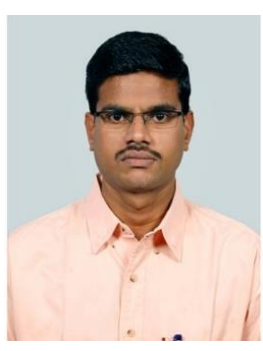

Ratna Babu Chekka received his B.Tech and M.Tech in Computer Science and Engineering from R.V.R. \& J.C.College of Engineering, Guntur. At present he is pursuing Ph.D. from Acharaya Nagarjuna University, Guntur. He is currently working as Associate Professor in the Department of Computer Science and Engineering at R.V.R. \& J.C. College of Engineering, Guntur. He has 10 years of teaching experience. His research areas of interest include Cryptography \& Network Security, Information Security, Computer Networks and Image Processing.

How to cite this paper: Sridhar Mandapati, Raveendra Babu Bhogapathi, Ratna Babu Chekka,"A Hybrid Algorithm for Privacy Preserving in Data Mining", International Journal of Intelligent Systems and Applications(IJISA), vol.5, no.8, pp.47-53, 2013. DOI: 10.5815/ijisa.2013.08.06 